\title{
ROJ
}

\section{Effectiveness and feasibility of concurrent chemoradiotherapy using simultaneous integrated boost- intensity modulated radiotherapy with and without induction chemotherapy for locally advanced pancreatic cancer}

\author{
Eun Sang Oh, MD', Tae Hyun Kim, MD', Sang Myung Woo, MD², Woo Jin Lee, MD², \\ Ju Hee Lee, MD², Sang Hee Youn, MD', Sung Sik Han, MD², Sang Jae Park, MD², \\ Dae Yong Kim, MD' \\ ${ }^{1}$ Proton Therapy Center, ${ }^{2}$ Center for Liver Cancer, National Cancer Center Research Institute and Hospital, \\ National Cancer Institute, Goyang, Korea
}

\begin{abstract}
Purpose: To evaluate the effectiveness and feasibility of chemoradiotherapy (CRT) using simultaneous integrated boost-intensity modulated radiotherapy (SIB-IMRT) in locally advanced pancreatic cancer (LAPC) patients.

Materials and Methods: Between January 2011 and May 2015, 47 LAPC patients received CRT using SIB-IMRT. Prior to SIBIMRT, 37 patients (78.7\%) received induction chemotherapy (IC-CRT group) and remaining 10 patients (21.3\%) did not received induction chemotherapy (CRT group). During SIB-IMRT, all patients received concomitant chemotherapy, with gemcitabine $(n=37)$ and capecitabine $(n=10)$.

Results: At the time of analysis, 45 patients had died and 2 patients remained alive and the median follow-up time was 14.2 months (range, 3.3 to 51.4 months). For all patients, the median times of local progression-free survival (LPFS), progression-free survival (PFS), and overall survival (OS) were 18.1, 10.3, and 14.2 months, respectively. The median time of LPFS between IC-CRT and CRT groups was similar (18.1 months vs. 18.3 months, $p=0.711$ ). IC-CRT group had a higher trend in PFS (10.9 months vs. 4.1 months, $p=0.054$ ) and had significantly higher OS (15.4 months vs. 9.5 months, $p=0.007)$ than CRT group. In multivariate analysis, the use of induction chemotherapy and tumor response were significant factors associated with $0 S(p<0.05$, each). During SIBIMRT, toxicity of grade $\geq 3$ was observed in 7 patients (14.9\%) in all patients.

Conclusions: CRT using SIB-IMRT is feasible and promising in LAPC patients.
\end{abstract}

Keywords: Pancreas neoplasms, Radiotherapy, Intensity-modulated radiotherapy, Survival

\section{Introduction}

Pancreatic cancer is the fifth cause of cancer-related mortality in Korea [1]. At the time of diagnosis, approximately $50 \%$ of patients present with metastatic disease and of the remaining patients with locoregional disease, about 70\% present with

Received 12 February 2018, Revised 15 May 2018, Accepted 23 July 2018.

Correspondence: Tae Hyun Kim, MD, Proton Therapy Center, National Cancer Center Research Institute and Hospital, National Cancer Institute, 323 Ilsan-ro, Ilsandong-gu, Goyang 10408, Korea. Tel: +82-31-920-1725, Fax: +82-31-920-0149, E-mail: k2onco@ncc.re.kr

(C) This is an Open Access article distributed under the terms of the Creative Commons Attribution Non-Commercial License (http://creativecommons.org/ licenses/by-nc/4.0/) which permits unrestricted non-commercial use, distribution, and reproduction in any medium, provided the original work is properly cited.

www.e-roj.org 


\section{Eun Sang Oh, et al}

locally advanced unresectable disease [2]. Historically, the prognosis for patients with locally advanced pancreatic cancer (LAPC) has remained poor due to high rates of local and distant tumor progression despite treatment with chemotherapy, radiotherapy, or both, and median survival with chemotherapy or chemoradiotherapy (CRT) is about 8-12 months [3,4]. Currently, treatment options for patients with LAPC include chemotherapy alone, CRT, and induction chemotherapy followed by CRT (IC-CRT) $[4,5]$. However, the role of radiotherapy in the treatment of these patients is disputed due to conflicting results of several randomized trials [6-11] and higher rates of toxicity $[7,10]$ and costs than chemotherapy alone. In patients with LAPC, distant metastases are a dominant cause of disease progression, but several autopsy series $[3,12]$ and one population-based study [13] showed that about $30 \%-40 \%$ of patients with LAPC die from locally destructive disease rather than distant metastasis. In addition, in the recently reported LAP07 trial [11], although the addition of radiotherapy to chemotherapy did not show a significant survival advantage, the patients treated with IC-CRT group showed a significantly reduced local tumor progression rate $(32 \%$ vs. $46 \%, p<0.05)$ and longer period without treatment ( 6.1 vs. 3.7 months, $p<0.05$ ) than those treated with chemotherapy alone. These findings support the hypothesis that CRT can improve survival and quality of life by decreasing local tumor progression.

With recent advances in planning and delivery of radiotherapy like intensity-modulated radiotherapy (IMRT), it can deliver a high dose to the tumor while reducing the dose to the surrounding normal tissues, such as gastrointestinal structures, and several studies have demonstrated that IMRT significantly lower rates of toxicities compared to treating with conventional (RT) [14-17]. Besides the conformal dose distribution, IMRT can exploit the potential biologic advantages of accelerated form of radiotherapy, known as simultaneous integrated boost-IMRT (SIB-IMRT), in which a higher dose can be delivered to the gross tumor volume (GTV) while a lower dose is delivered to areas of subclinical disease at the same time. The potential advantage of this accelerated fractionation is to improve tumor control by reducing the accelerated repopulation of tumor clonogenic cells by shortening overall treatment time. On this background, the patients with LAPC have been treated at our institution by RT using the SIB-IMRT technique since January 2011. This study was designed to retrospectively analyze the clinical outcomes of SIB-IMRT in LAPC patients and to evaluate the clinical effectiveness and safety of this method.

\section{Materials and Methods}

\section{Patients}

This study included consecutive 47 patients with LAPC, histologically confirmed with adenocarcinoma, who received CRT using SIB-IMRT between January 2011 and May 2015. Prior to the treatment, all patients were given physical examinations and had complete blood counts, liver function test, serum carbohydrate antigen 19-9 (CA 19-9) concentration, chest radiography, and computed tomography (CT) of the abdomen and pelvis and/or positron-emission tomography (PET). All tumors were staged using the American Joint Committee on Cancer (AJCC), 7th edition, and were classified as stage CT4 (unresectable disease), based on the CT scans, with tumor extension to the celiac axis or superior mesenteric artery. Patients with borderline resectable pancreatic cancer were not included in this study. Lymph node involvement was defined by the presence of a lymph node of at least $1 \mathrm{~cm}$ in the short axis with a spiculated or indistinct border. Patient characteristics are summarized in Table 1. The study was performed in accordance with the guidelines of our Institutional Review Board (No. NCC20110567), which deemed that informed consent was not required because the study was retrospective.

\section{Treatment}

For SIB-IMRT planning, contrast-enhanced four-dimensional CT images were acquired, with $2.5 \mathrm{~mm}$ slice thicknesses, under shallow respiration using a four-dimensional CT simulator (LightSpeed RT; GE Healthcare, Waukesha, WI, USA). The acquired $\mathrm{CT}$ images were reconstructed in 10 equally spaced respiratory phases and, in the post-processing stage, average intensity projection (AIP) CT images were reconstructed. All $\mathrm{CT}$ images were transferred to a treatment planning system (Eclipse v8.0; Varian Medical System, Palo Alto, CA, USA) and contours for targets and organs at risk (OARs) were drawn. The GTV included all detectable primary tumors as determined by AIP-CT images and an internal target volume (ITV) was obtained by summing the GTVs of all respiratory motion phases. The clinical target volume (CTV) included the ITV and the volumes of regional lymph nodes, including pericholedochal, celiac, and pancreaticoduodenal nodes. The planning target volumes 1 and 2 (PTV1 and PTV2) included the ITV plus 3-5 mm margin and CTV plus 5-7 $\mathrm{mm}$ margin in all directions, respectively. SIB-IMRT planning was performed using five coplanar or non-coplanar beams of $6 \mathrm{MV}$ photons. The treatment was designed so that at least 95\% of the PTV would receive $100 \%$ of the prescribed dose, and such that 
Table 1. Patients' characteristics

\begin{tabular}{|c|c|c|c|c|}
\hline Characteristic & Total & $\mathrm{IC}-\mathrm{CRT}^{\mathrm{a})}(\mathrm{n}=37)$ & CRT $(n=10)$ & $p$-value \\
\hline \multicolumn{5}{|l|}{ Sex } \\
\hline Male & $25(52.2)$ & $20(54.1)$ & $5(50.0)$ & $1.000^{c)}$ \\
\hline Female & $22(46.8)$ & $17(45.9)$ & $5(50.0)$ & \\
\hline Age (yr) & $67(45-92)$ & $67(45-76)$ & $73(51-92)$ & $0.244^{\mathrm{d})}$ \\
\hline$<70$ & $28(59.6)$ & $24(64.9)$ & $4(40.0)$ & $0.276^{c)}$ \\
\hline$\geq 70$ & $19(40.4)$ & $13(35.1)$ & $6(60.0)$ & \\
\hline \multicolumn{5}{|l|}{ ECOG performance scale } \\
\hline 0 & $38(80.9)$ & $29(78.4)$ & $9(90.0)$ & $0.660^{c)}$ \\
\hline 1 & $9(19.1)$ & $8(21.6)$ & $1(10.0)$ & \\
\hline \multicolumn{5}{|l|}{ Tumor location } \\
\hline Head/neck & $26(55.3)$ & $20(54.1)$ & $6(60.0)$ & $1.000^{c)}$ \\
\hline Body/tail & $21(44.7)$ & $17(45.9)$ & $4(40.0)$ & \\
\hline Tumor size $(\mathrm{cm})$ & $3.8(1.5-7.2)$ & $3.6(1.5-7.2)$ & $3.9(2.6-7.2)$ & $0.289^{d)}$ \\
\hline$\leq 4$ & $32(68.1)$ & $26(70.3)$ & $6(60.0)$ & $0.704^{c)}$ \\
\hline$>4$ & $15(31.9)$ & $11(29.7)$ & $4(40.0)$ & \\
\hline \multicolumn{5}{|l|}{ cN classification } \\
\hline No & $22(46.8)$ & $19(51.4)$ & $3(30.0)$ & $0.297^{c)}$ \\
\hline N1 & $25(53.2)$ & $18(48.6)$ & $7(70.0)$ & \\
\hline Pretreatment CA 19-9 level (U/mL) & $146(2-5,275)$ & $69.2(2-3,334)$ & $280(5-5,275)$ & $0.373^{d)}$ \\
\hline$\leq 100$ & $21(44.7)$ & $19(51.4)$ & $2(20.0)$ & $0.150^{c)}$ \\
\hline$>100$ & $26(55.3)$ & $18(48.6)$ & $8(80.0)$ & \\
\hline CA 19-9 percent decrease (\%) & $36.3(-613.4-91.2)$ & $36.5(-337.9-91.2)$ & $23.8(-613.4-85.7)$ & $0.236^{d)}$ \\
\hline$\leq 50$ & $30(63.8)$ & $24(64.9)$ & $6(60.0)$ & $1.000^{c)}$ \\
\hline$>50$ & $17(36.2)$ & $13(35.1)$ & $4(40.0)$ & \\
\hline \multicolumn{5}{|l|}{ Concurrent chemotherapy } \\
\hline Gemcitabine & $37(78.7)$ & $36(97.3)$ & $1(10.0)$ & $<0.001^{c)}$ \\
\hline Capecitabine & $10(21.3)$ & $1(2.7)$ & $9(90.0)$ & \\
\hline \multicolumn{5}{|l|}{ Maintenance chemotherapy } \\
\hline No & $24(51.5)$ & $17(45.9)$ & $7(70.0)$ & $0.286^{c)}$ \\
\hline Yes & $23(48.9)$ & $20(54.1)$ & $3(30.0)$ & \\
\hline \multicolumn{5}{|l|}{ Post-CRT surgery } \\
\hline No & $35(74.5)$ & $27(73.0)$ & $8(80.0)$ & $1.000^{c)}$ \\
\hline Yes & $12(25.5)$ & $10(27.0)$ & $2(20.0)$ & \\
\hline
\end{tabular}

Values are presented as number (\%) or median (range).

IC-CRT, induction chemotherapy followed by concurrent chemoradiotherapy; CRT, concurrent chemoradiotherapy; ECOG Eastern Cooperative Oncology Group; CA 19-9, carbohydrate antigen 19-9.

CA 19-9 percent decrease $(\%)=\frac{(\text { Pre-CRT }- \text { Post-CRT) }}{\text { Pre-CRT }} \times 100$.

${ }^{a}$ Gemcitabine + cisplatin $(n=33)$, gemcitabine + erlotinib $(n=2)$, gemcitabine + capecitabine $(n=1)$, and capecitabine + oxaliplatin $(n=1)$.

${ }^{\text {b) }}$ Maximum diameter of the primary tumor. ${ }^{c}$ Fisher exact test, two-tailed. ${ }^{d)}$ t-test, two-tailed.

a contiguous volume of no more than $2 \mathrm{~cm}^{3}$ inside the PTV would receive no more than $125 \%$ of the prescribed dose. The equivalent dose in 2 Gy fraction $\left(\mathrm{EOD}_{2}, \mathrm{~Gy} \mathrm{y}_{10}\right)$, calculated using a linear quadratic model with $\alpha / \beta$ ratios of 10 for acute effects on tumor and OARs, was used for normal tissue constraints. The maximum dose to the spinal cord could not exceed 45 $\mathrm{Gy}_{10}$; the relative volumes of the total liver that received doses of $30 \mathrm{~Gy}_{10}$ were below 50\%; the absolute volumes of the esophagus and stomach that received at least $55 \mathrm{~Gy}_{10}$ were $\leq 2$ $\mathrm{cm}^{3} ;$ and the absolute volumes of the small and large bowel that received at least $50 \mathrm{~Gy}_{10}$ were $\leq 2 \mathrm{~cm}^{3}$. The prescribed doses to PTV1 and PTV2 were $55 \mathrm{~Gy}\left(\mathrm{EQD}_{2}=57.3 \mathrm{~Gy}_{10}\right)$ and $44 \mathrm{~Gy}\left(\mathrm{EQD}_{2}=44 \mathrm{~Gy}_{10}\right)$ in 22 fractions, 5 fractions/week, respectively [18]. At each treatment fraction, digital orthogonal fluoroscopy was used to position the patient and to verify the isocenter.

Prior to SIB-IMRT, 37 patients (78.7\%) received induction chemotherapy (IC-CRT group) and remaining 10 patients 


\section{Eun Sang Oh, et al}

(21.3\%) did not received induction chemotherapy (CRT group). IC-CRT group received a median of 2 cycles (range, 2 to 6 cycles) of induction chemotherapy, with gemcitabine and cisplatin $(n=33)$, gemcitabine and erlotinib $(n=2)$, gemcitabine and capecitabine $(n=1)$, and capecitabine and oxaliplatin $(n=1)$.

During SIB-IMRT, all patients received concomitant chemotherapy, with gemcitabine $(n=37)$ and capecitabine ( $n$ = 10) (Table 1). After completion of SIB-IMRT, patients who had resectable disease were considered for surgical resection, whereas patients who still had unresectable disease were considered for maintenance chemotherapy until disease progression or treatment-limiting toxicity. Chemotherapy regimens were chosen by physician preference and patients who refused further chemotherapy or had poor performance status received supportive care.

\section{Follow-up and statistical analysis}

Patients were assessed weekly during SIB-IMRT and after completion of SIB-IMRT at 1 month, then every 2 to 3 months for the first 3 years and every 6 months thereafter. Followup evaluations consisted of a physical examination, complete blood count, liver function test, measurement of serum CA 19-9, chest radiography and CT scan of the abdomen and pelvis. The responses of the primary tumor were defined as the maximal tumor response observed during the follow-up period unless progression occurred, which was determined by comparing CT scans before and after SIB-IMRT using the Response Evaluation Criteria in Solid Tumors criteria (RECIST v1.1). Objective response rates were calculated as the rate of complete response (CR) and partial response (PR). Patients with CR or PR were considered 'Responders', and those with stable disease (SD) or progressive disease (PD) were considered 'Nonresponders'. Toxicity was recorded according to the National Cancer Institute - Common Terminology Criteria for Adverse Events (v4.03).

Recurrence was proven pathologically by biopsy or cytology and/or radiological findings showing an increase in size over time. Local failure was defined as progression of the primary tumor or recurrence at the primary tumor bed, regional failure was defined as progression or recurrence of disease in regional lymph nodes and soft tissues located near the primary tumor, while distant failure was defined as the development of distant metastasis. Locoregional progression-free survival (LPFS), relapse-free survival (RFS), and overall survival (OS) were defined as the intervals the commencement of induction chemotherapy or CRT to the date of detection of locoregional
Radiation Oncology Journal ROJ

progression, any detection of relapse, and death or last follow-up, respectively. The OS rates were calculated using the Kaplan-Meier method. Univariate analysis of parameters predicting OS were assessed with log rank tests, followed by multivariate analysis using Cox proportional hazard model with a stepwise forward procedure containing factors of $p<$ 0.1 in univariate analysis. All statistical analyses were twosided and were performed using STATA software (version 14.0; StataCorp., College Station, TX, USA). The p-values less than 0.05 were considered statistically significant.

\section{Results}

Patient characteristics are summarized in Table 1. The patients in CRT group had a trend in higher frequencies of old age ( $\geq 70$ years), larger tumor size ( $>4 \mathrm{~cm})$, lymph node positive, and high pretreatment CA 19-9 level (>100 U/mL), than the patients in IC-CRT group, but their differences were not significant ( $p<0.05$, each). During SIB-IMRT, the patients in CRT group more frequently received concomitant chemotherapy with capecitabine $(90 \%$ vs. $3 \%, p<0.05)$ than the patient in IC-CRT group (Table 1). Tumor responses for all patients, IC-CRT group, and CRT group were as follows: PR in 29 (61.7\%), 23 (62.2\%), and $6(60 \%)$; SD in $18(38.3 \%), 14(37.8 \%)$, and $4(40 \%)(p=$ 0.589). The best response after SIB-IMRT were 7.8 months. After completion of SIB-IMRT, 12 (25.5\%) patients with prominent tumor regression subsequently underwent surgical resection, while 10 of them (83.3\%) achieved Ro resection: 10 of 37 patients (27\%) in IC-CRT group and 2 of 10 patients (20\%) in CRT group underwent surgical resection, respectively $(p<0.05)$ (Table 1). After completion of SIB-IMRT, 23 patients $(48.9 \%)$ received gemcitabine based chemotherapy until disease progression, treatment-limiting toxicity, or death and the remaining 24 patients (51.1\%) did not receive maintenance chemotherapy because of patient refusal or poor performance status. IC-CRT group more frequently received maintenance chemotherapy than CRT group (20/47 [54.1\%] vs. 3/10 [30\%]), but its difference was not significant $(p=0.286)$ (Table 1$)$.

At the time of analysis, 45 patients had died and 2 patients remained alive and the median follow-up time in all patients was 14.2 months (range, 3.3 to 51.4 months). Of 47 patients, 44 (93.6\%) developed disease progression, including 15 (31.9\%) with local progression, 7 (14.9\%) with regional progression, and $40(85.1 \%)$ with distant metastases (Fig. 1). There were no differences in the distributions of locoregional progression $(18 / 37$ [48.6\%] vs. $3 / 10$ [30\%], $p=0.301)$ and distant metastasis $(32 / 37$ [86.5\%] vs. $8 / 10[80 \%], p=0.618)$ between 
A

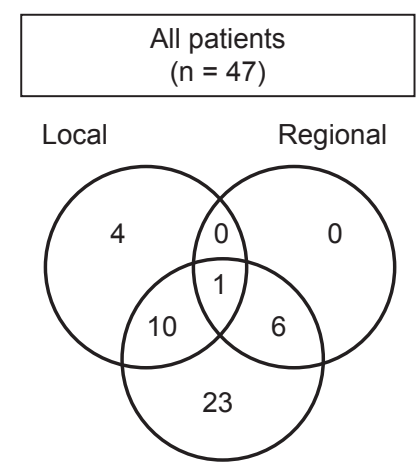

B

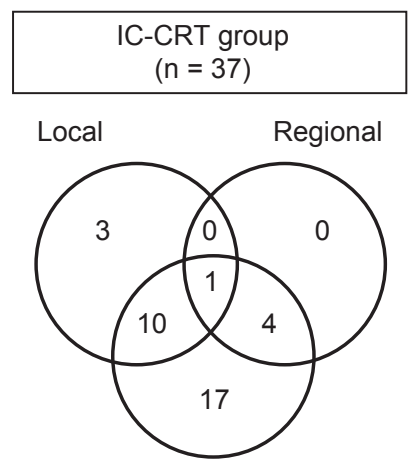

C Distant

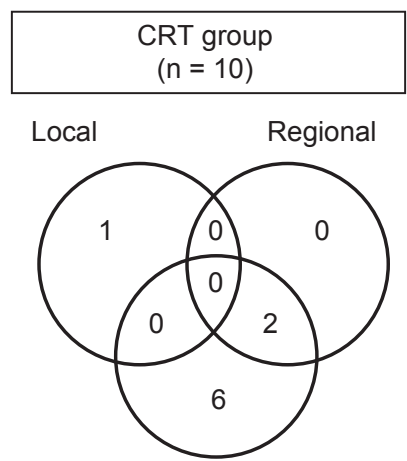

Fig. 1. Patterns of failure in all patients (A), induction chemotherapy followed by chemoradiotherapy (IC-CRT) group (B), and CRT without induction chemotherapy (CRT) group (C).

IC-CRT group and CRT group. The median times of LPFS, RFS, and $\mathrm{OS}$ in all patients were 18.1 months (95\% confidence interval [Cl], 15.1-21.2), 10.3 months (95\% Cl, 8.6-11.9), and 14.2 months (95\% Cl, 10.7-17.7), respectively.

Univariate and multivariate analyses were performed to identify parameters predicting OS (Table 2). In univariate analysis, tumor size, tumor response, and the use of induction chemotherapy were found to be significantly associated with longer OS. The patients with pretreatment CA 19-9 level of $\leq 100 \mathrm{U} / \mathrm{mL}$, concurrent chemotherapy with gemcitabine, surgery after chemoradiotherapy, and the use of maintenance chemotherapy had a trend of higher OS than those with pretreatment CA 19-9 level of $>100 \mathrm{U} / \mathrm{mL}$, concurrent chemotherapy with capecitabine, no surgery after chemoradiotherapy, and no use of maintenance chemotherapy, but their differences were not statistically significant ( $p>$ 0.05) (Table 2). In multivariate analysis, the use of induction chemotherapy and tumor response were significant factors associated with OS ( $p<0.05$, each) (Table 2).

The median LPFS between IC-CRT and CRT groups was similar (18.1 months vs. 18.3 months, $p=0.711$ ) (Fig. 2A). IC-CRT group had a trend towards longer RFS (10.9 months vs. 4.1 months, $p=0.054$ ) and had significantly higher OS (15.4 months vs. 9.5 months, $p=0.007$ ) than CRT group (Fig. $2 B, 2 C)$. To avoid the patient selection effect of induction chemotherapy by excluding the patients with occult metastasis, LPFS, RFS, and OS between IC-CRT $(n=37)$ and CRT groups ( $n=7$ ), excluding 3 patients who developed early distant metastasis within 3 months after SIB-IMRT, were compared (Fig. 2D-2F). Similarly, median times of LPFS between IC-CRT and CRT group were similar (18.1 months vs. 18.3 months, $p=0.563)$. IC-CRT group had a higher trend in longer RFS (10.9 months vs. 7.3 months) and OS (15.4 months vs. 9.6 months) than CRT group, but their differences were not significant ( $p>0.05$, each). This result may be due to the small number of patients in CRT group $(n=7)$.

During SIB-IMRT, toxicity of grade 3 was observed in 7 (14.9\%) patients in all patients and hematologic toxicities of grade 3 were significantly higher in IC-CRT group than in CRT group (16\% [6/37] vs. 0\% [0/10], $p=0.009$ ) (Table 3). After completion of the SIB-IMRT, toxicities of grade $\leq 2$ and 3 toxicities in IC-CRT and CRT group were observed in 10 (27\%) and $0(0 \%), 3(30 \%)$ and $1(10 \%)$, respectively, and there were no significant differences ( $p>0.05$, each). No treatmentrelated toxicities of grade $\geq 4$ were observed (Table 3 ).

\section{Discussion and Conclusion}

Combinations of local and systemic treatments, such as IC-CRT, CRT, have been tried in LAPC patients due to high risk of both local and distant progression $[3,4,6-11,16,19]$. When considering the combinations of radiotherapy and chemotherapy in LAPC patients, its effects of local and distant tumor control as well as its tolerability might be important. Previous studies which treated LAPC patients with CRT using conventional RT reported $27 \%-77 \%$ of grade $\geq 3$ toxicity and median OS duration of 8-12 months $[3,4,6-11,19,20]$. Recently, IMRT technique has been applied in LAPC patients to improve local tumor control by delivering the higher dose to tumor and to minimize potential of toxicity by reducing radiation to surrounding normal tissues, such as adjacent gastrointestinal structures, and several studies have shown lower rate (15\%-24\%) of grade $\geq 3$ toxicity and promising median OS (12-15.3 months) [14-17,21]. Similarly, in present study, we applied CRT using SIB-IMRT with/without induction chemotherapy for LAPC patients and observed a median OS of 
Table 2. Univariate and multivariate analysis of clinical characteristics associated with overall survival

\begin{tabular}{|c|c|c|c|c|}
\hline \multirow[t]{3}{*}{ Characteristic } & \multicolumn{4}{|c|}{ Overall survival (mo) } \\
\hline & \multicolumn{2}{|c|}{ Univariate } & \multicolumn{2}{|c|}{ Multivariate } \\
\hline & Median (95\% CI) & $p$-value & HR (95\% Cl) & $p$-value ${ }^{b)}$ \\
\hline Age (yr) & & 0.367 & & - \\
\hline$<70$ & $15.0(12.0-18.0)$ & & - & \\
\hline$\geq 70$ & $12.4(9.1-15.7)$ & & - & \\
\hline Sex & & 0.287 & & - \\
\hline Male & $14.2(11.8-16.6)$ & & - & \\
\hline Female & $12.3(5.5-19.1)$ & & - & \\
\hline Pretreatment CA 19-9 level (U/mL) & & 0.261 & & - \\
\hline$\leq 100$ & $18.9(13.2-24.6)$ & & - & \\
\hline$>100$ & $12.3(9.9-14.7)$ & & - & \\
\hline CA 19-9 percent decrease (\%) & & 0.613 & & - \\
\hline$\leq 50$ & $17.9(11.9-23.9)$ & & - & \\
\hline$>50$ & $12.4(9.7-15.2)$ & & - & \\
\hline Tumor location & & 0.861 & & - \\
\hline Head/neck & $13.5(8.4-18.6)$ & & - & \\
\hline Body/tail & $14.2(10.5-17.9)$ & & - & \\
\hline Tumor size $(\mathrm{cm})$ & & 0.018 & & - \\
\hline$\leq 4$ & $15.4(12.2-18.6)$ & & - & \\
\hline$>4$ & $11.5(10.4-12.6)$ & & - & \\
\hline cN classification & & 0.057 & & - \\
\hline No & $15.0(8.1-21.9)$ & & - & \\
\hline N1 & $12.4(10.1-14.7)$ & & - & \\
\hline Concurrent chemotherapy & & 0.103 & & - \\
\hline Gemcitabine & $15.0(12.3-17.7)$ & & - & \\
\hline Capecitabine & $9.6(7.1-12.1)$ & & - & \\
\hline Tumor response & & 0.002 & & 0.002 \\
\hline Responder & $17.2(10.3-24.1)$ & & 1.000 & \\
\hline Non-responder & $10.9(9.0-12.8)$ & & $3.330(1.574-7.047)$ & \\
\hline Induction chemotherapy & & 0.007 & & 0.026 \\
\hline No & $9.5(6.4-12.6)$ & & 1.000 & \\
\hline Yes & $15.4(11.8-19.0)$ & & $0.050(0.004-0.700)$ & \\
\hline Post-CRT surgery & & 0.129 & & - \\
\hline No & $12.3(9.2-15.4)$ & & - & \\
\hline Yes & $15.4(11.5-19.3)$ & & - & \\
\hline Maintenance chemotherapy & & 0.218 & & - \\
\hline No & $11.6(7.8-15.4)$ & & - & \\
\hline Yes & $17.2(11.4-23.0)$ & & - & \\
\hline
\end{tabular}

Cl, confidence interval; CA 19-9, carbohydrate antigen 19-9; Responder, complete or partial response; Non-responder, stable disease or progressive disease; $\mathrm{CRT}$, concurrent chemoradiotherapy.

${ }^{\text {a) } L o g}$ rank test. ${ }^{\text {b) }}$ Cox proportional hazards model.

14.2 months and $14.9 \%$ of grade 3 toxicity, without grade $\geq 4$ toxicity. Although direct comparison of data against previous studies $[3,4,6-11,16,17,19,21]$ and present study is difficult due to heterogeneities of study population, such as performance status, tumor burden, and the various agents and sequence of chemotherapy administered, etc., median OS and incidence of grade $\geq 3$ toxicity in the present study were at the higher and lower end of the wide range reported previously, respectively.

As 30\% of LAPC patients have occult metastatic disease at diagnosis $[3,4,22]$, induction chemotherapy can help to select a subgroup of patients without early metastatic course who can potentially benefit from locoregional treatment, i.e., CRT. Several retrospective studies have shown a survival benefit of CRT after induction chemotherapy compared to chemotherapy or CRT alone [19,23-25]. Although recent LAP07 trial [11] did not show an OS benefit by addition of CRT after induction chemotherapy compared to chemotherapy alone, it showed a significant decrease in rate of local progression 
A

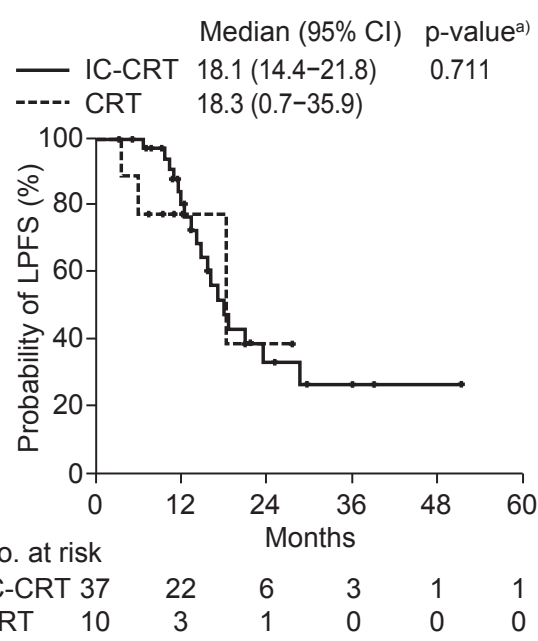

B

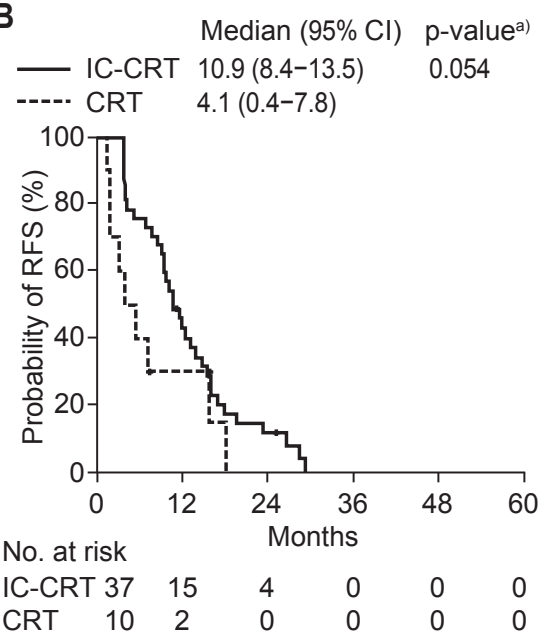

C

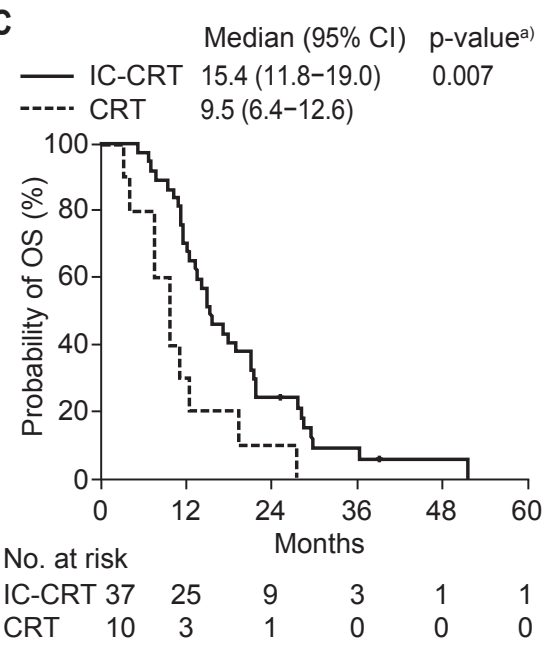

D

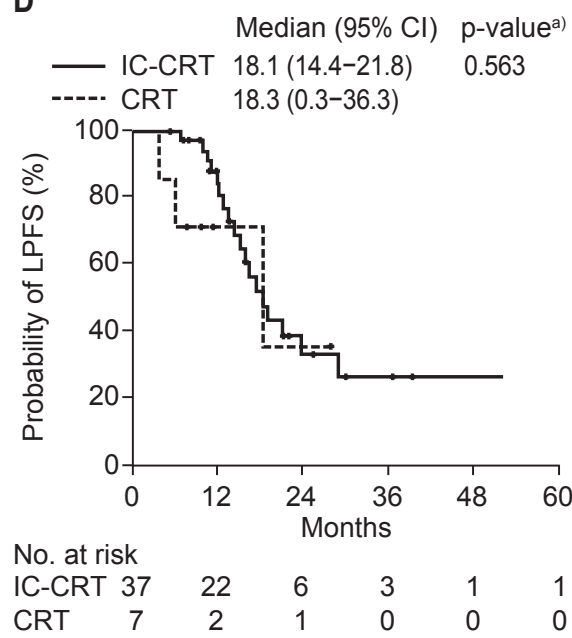

E

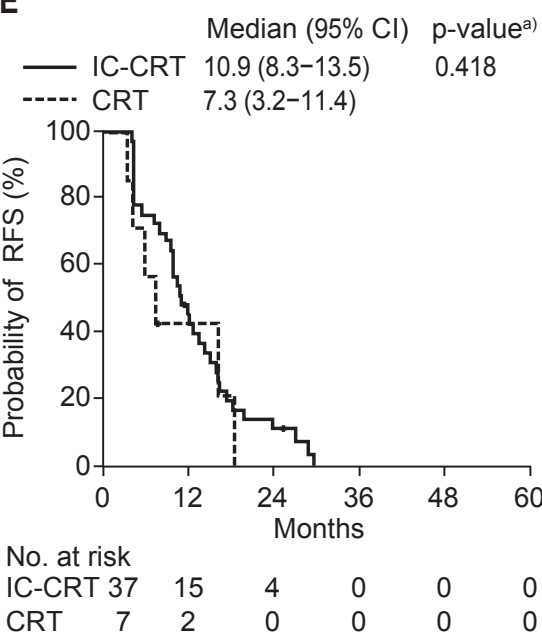

$F$

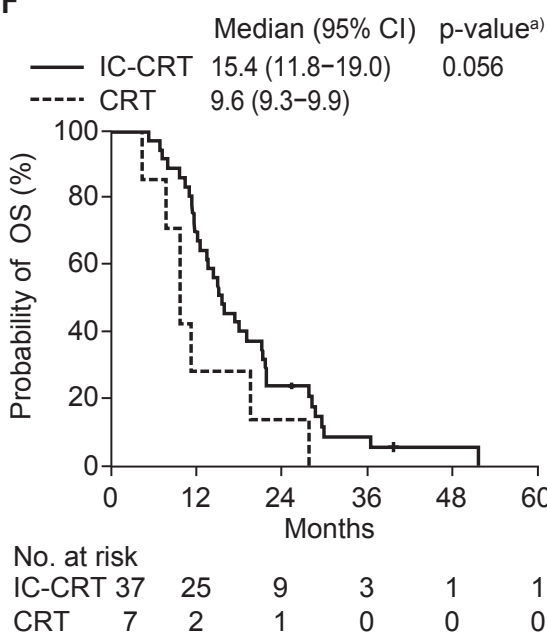

Fig. 2. (A) Local progression-free survival (LPFS), (B) relapse-free survival (RFS), and (C) overall survival (OS) curves in all patients according to treatment groups. (D) LPFS, (E) RFS, and (F) OS in patients without distant metastasis within 3 months after treatment according to treatment groups. IC-CRT, induction chemotherapy followed by chemoradiotherapy; CRT, chemoradiotherapy without induction chemotherapy; $\mathrm{Cl}$, confidence interval. ${ }^{\text {al } L o g}$ rank test.

in IC-CRT compared to chemotherapy alone. Recent cohort studies also showed that CRT after multi-agent induction chemotherapy or longer duration, i.e., at least 3 cycles, of induction chemotherapy had longer OS than chemotherapy alone $[25,26]$. Similarly, in present study, the patients in ICCRT had significantly longer median OS time than those in CRT group (15.4 months vs. 9.5 months, $p=0.007$ ). In addition, IC-CRT group had trend longer OS than CRT group, after excluding the patients who developed early distant metastasis within 3 months after CRT. These findings suggest that induction chemotherapy can select a biologically favorable group without early progression of distant metastasis who can benefit from locoregional treatment.

The conversion to resectability and actual down staging of tumor is one of ultimate goal of treatment in LAPC patients, but actual rate of surgical resection after CRT using conventional RT was uncommon (about 4\%) in previous randomized trials $[9,11]$. Recently, in a phase $1 / / 1$ trial in LAPC patients treated with CRT using IMRT with dose escalation from 50-60 Gy in 25 fractions, Ben-Josef et al. [17] reported $24 \%$ resection rate, while $83 \%$ of them achieved R0 resection. Huguet et al. [21] also reported 19\% resection rate, while 85\% of them achieved R0 resection in LAPC patients treated with induction chemotherapy followed by CRT using SIB- 


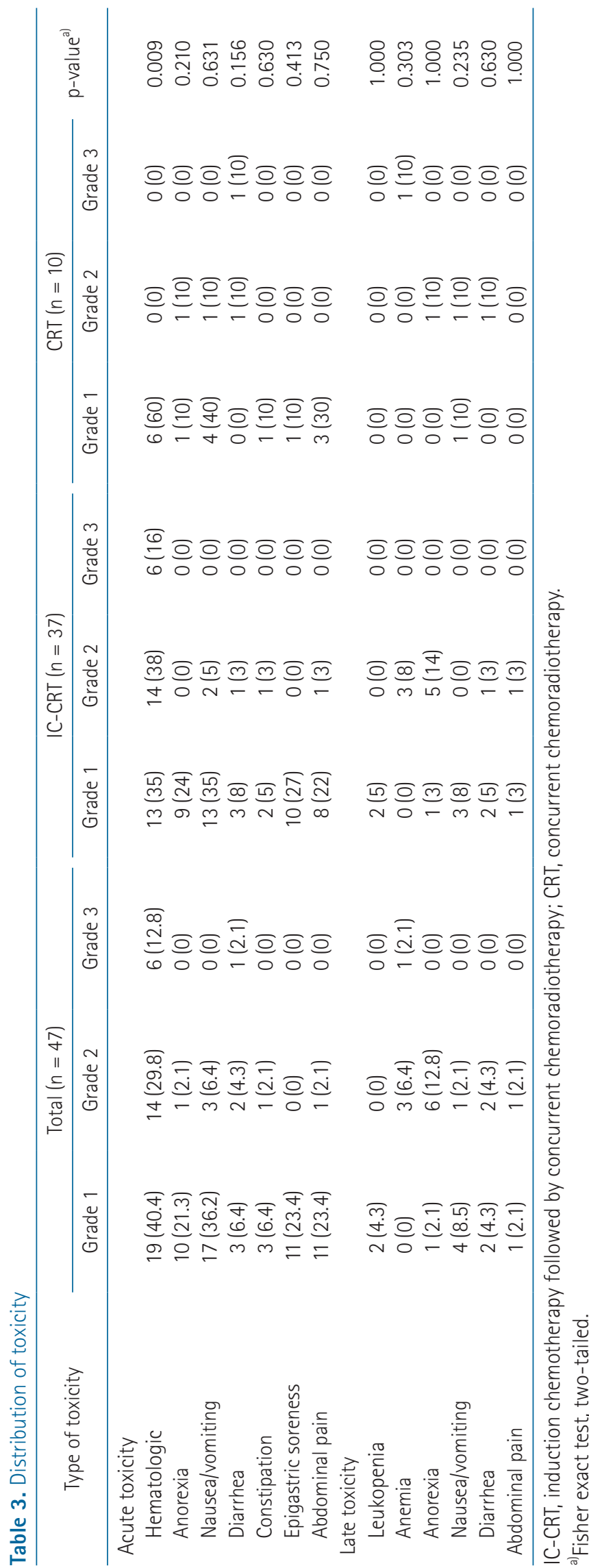

IMRT. Similarly, in present study, 12 patients (25.5\%) underwent surgical resection after CRT using SIB-IMRT with/without induction chemotherapy and 10 of them (83\%) achieved R0 resection. Although OS between the patients who underwent surgical resection after SIB-IMRT and those who did not was not significantly different due to the small number of patients who underwent surgical resection $(n=12)$, the patients who underwent surgical resection had a trend towards higher median OS than those who did not undergo surgical resection (15.4 months vs. 12.3 months, $p=0.129$ ) (Table 2) and one patient who achieved complete response after surgery remained alive at 51.4 months. These findings suggest that CRT using SIB-IMRT might provide higher resection rate and subsequently increase the probability of cure in LAPC patients.

This study was retrospective and thus had certain inherent limitations. First, our data were from a single institutional study with a relatively small and heterogeneity of various chemotherapeutic agents and sequence; thus, the effects of systemic chemotherapy and probable selection bias were not thoroughly evaluated. Second, this study included LAPC patients treated with CRT and did not include the patients treated with chemotherapy alone, and thus we could not compare CRT with/without induction chemotherapy with chemotherapy alone. Third, CRT group had a trend towards higher frequencies of several unfavorable factors, such as old age, large tumor size, lymph node positive, and high pretreatment CA 19-9 level, and less frequently received maintenance chemotherapy than the patients in IC-CRT group. Thus, interpretations of our data should be careful. Lastly, the assessment of toxicity in a retrospective analysis might have underestimated risks due to incomplete reporting in clinic notes and recall bias. Despite these limitations, SIB-IMRT has a potential advantage, such as increasing dose to target and minimising RT dose to surrounding gastrointestinal structures, compared to conventional RT, and thus further large-scale prospective studies including combinations of SIB-IMRT and modern systemic chemotherapy regimens $[27,28]$, such as 5 -fluorouracil, irinotecan plus oxaliplatin and gemcitabine plus nabpaclitaxel, is warranted.

In conclusion, our data showed that CRT using SIBIMRT for LAPC patients showed promising results, such as median OS time of 14.2 months and grade $\geq 3$ toxicity 
of $14.9 \%$, and suggested that SIB-IMRT could be feasible and promising as one component of combined treatment modalities for LAPC patients.

\section{Conflict of Interest}

No potential conflict of interest relevant to this article was reported.

\section{Acknowledgments}

This study was supported by National Cancer Center Grant (No. NCC1710030).

\section{References}

1. Jung KW, Won $Y J$, Kong HJ, et al. Cancer statistics in Korea: incidence, mortality, survival, and prevalence in 2012. Cancer Res Treat 2015;47:127-41.

2. Sener SF, Fremgen A, Menck HR, Winchester DP. Pancreatic cancer: a report of treatment and survival trends for 100,313 patients diagnosed from 1985-1995, using the National Cancer Database. J Am Coll Surg 1999;189:1-7.

3. lacobuzio-Donahue CA, Fu B, Yachida S, et al. DPC4 gene status of the primary carcinoma correlates with patterns of failure in patients with pancreatic cancer. J Clin Oncol 2009;27:1806-13.

4. Ben-Josef $E_{1}$ Lawrence TS. Chemoradiotherapy for unresectable pancreatic cancer. Int J Clin Oncol 2008;13:121-6.

5. Tempero MA, Malafa MP, Al-Hawary M, et al. Pancreatic adenocarcinoma, Version 2.2017, NCCN Clinical Practice Guidelines in Oncology. J Natl Compr Canc Netw 2017;15:1028-61.

6: Hazel JJ, Thirlwell MP, Huggins M, Maksymiuk A, MacFarlane JK. Multi-drug chemotherapy with and without radiation for carcinoma of the stomach and pancreas: a prospective randomized trial. J Can Assoc Radiol 1981;32:164-5.

7. Klaassen DJ, Maclntyre JM, Catton GE, Engstrom PF, Moertel CG. Treatment of locally unresectable cancer of the stomach and pancreas: a randomized comparison of 5-fluorouracil alone with radiation plus concurrent and maintenance 5-fluorouracil: an Eastern Cooperative Oncology Group study. J Clin Oncol 1985;3:373-8.

8. Gastrointestinal Tumor Study Group. Treatment of locally unresectable carcinoma of the pancreas: comparison of combined-modality therapy (chemotherapy plus radiotherapy) to chemotherapy alone. J Natl Cancer Inst 1988;80:751-5.
9. Chauffert B, Mornex F, Bonnetain F, et al. Phase III trial comparing intensive induction chemoradiotherapy (60 Gy, infusional 5-FU and intermittent cisplatin) followed by maintenance gemcitabine with gemcitabine alone for locally advanced unresectable pancreatic cancer. Definitive results of the 2000-01 FFCD/SFRO study. Ann Oncol 2008;19:1592-9.

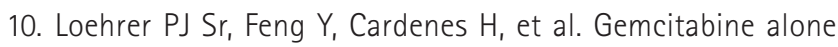
versus gemcitabine plus radiotherapy in patients with locally advanced pancreatic cancer: an Eastern Cooperative Oncology Group trial. J Clin Oncol 2011;29:4105-12.

11. Hammel $P$, Huguet $F$, van Laethem $J L$, et al. Effect of chemoradiotherapy vs chemotherapy on survival in patients with locally advanced pancreatic cancer controlled after 4 months of gemcitabine with or without erlotinib: the LAP07 Randomized Clinical Trial. JAMA 2016;315:1844-53.

12. Kamisawa $T$, Isawa $T$, Koike $M$, Tsuruta $K$, Okamoto $A$. Hematogenous metastases of pancreatic ductal carcinoma. Pancreas 1995;11:345-9.

13. Peixoto RD, Speers C, McGahan CE, Renouf DJ, Schaeffer DF, Kennecke HF. Prognostic factors and sites of metastasis in unresectable locally advanced pancreatic cancer. Cancer Med 2015:4:1171-7.

14. Yovino S, Poppe M, Jabbour S, et al. Intensity-modulated radiation therapy significantly improves acute gastrointestinal toxicity in pancreatic and ampullary cancers. Int J Radiat Oncol Biol Phys 2011;79:158-62.

15. Bittner MI, Grosu AL, Brunner TB. Comparison of toxicity after IMRT and 3D-conformal radiotherapy for patients with pancreatic cancer: a systematic review. Radiother Oncol 2015;114:117-21.

16. Krishnan S, Chadha AS, Suh Y, et al. Focal radiation therapy dose escalation improves overall survival in locally advanced pancreatic cancer patients receiving induction chemotherapy and consolidative chemoradiation. Int J Radiat Oncol Biol Phys 2016;94:755-65.

17. Ben-Josef E, Schipper M, Francis IR, et al. A phase I/II trial of intensity modulated radiation (IMRT) dose escalation with concurrent fixed-dose rate gemcitabine (FDR-G) in patients with unresectable pancreatic cancer. Int J Radiat Oncol Biol Phys 2012;84:1166-71.

18. Kim TH, Park JW, Kim YJ, et al. Simultaneous integrated boost-intensity modulated radiation therapy for inoperable hepatocellular carcinoma. Strahlenther Onkol 2014;190:88290.

19. Huguet $F$, Andre T, Hammel P, et al. Impact of chemoradiotherapy after disease control with chemotherapy in locally advanced pancreatic adenocarcinoma in GERCOR 
Eun Sang Oh, et al

phase II and III studies. J Clin Oncol 2007;25:326-31.

20. Bae JS, Kim DH, Kim WT, Kim YH, Park D, Ki YK. The role of surgical clips in the evaluation of interfractional uncertainty for treatment of hepatobiliary and pancreatic cancer with postoperative radiotherapy. Radiat Oncol J 2017;35:65-70.

21. Huguet $F$, Hajj $C$, Winston $C B$, et al. Chemotherapy and intensity-modulated radiation therapy for locally advanced pancreatic cancer achieves a high rate of R0 resection. Acta Oncol 2017:56:384-90.

22. Yoo T, Lee WJ, Woo SM, et al. Pretreatment carbohydrate antigen 19-9 level indicates tumor response, early distant metastasis, overall survival, and therapeutic selection in localized and unresectable pancreatic cancer. Int J Radiat Oncol Biol Phys 2011;81:e623-30.

23. Krishnan $S$, Rana $V$, Janjan NA, et al. Induction chemotherapy selects patients with locally advanced, unresectable pancreatic cancer for optimal benefit from consolidative chemoradiation therapy. Cancer 2007;110:47-55.

24. Li D, O'Reilly EM. Adjuvant and neoadjuvant therapy for pancreatic cancer. Surg Oncol Clin N Am 2016;25:311-26.

25. Torgeson A, Lloyd $S$, Boothe $D$, et al. Multiagent induction chemotherapy followed by chemoradiation is associated with improved survival in locally advanced pancreatic cancer. Cancer 2017;123:3816-24.

26. Faisal $F$, Tsai $H L$, Blackford $A$, et al. Longer course of induction chemotherapy followed by chemoradiation favors better survival outcomes for patients with locally advanced pancreatic cancer. Am J Clin Oncol 2016;39:18-26.

27. Conroy $T$, Desseigne $F$, Ychou $M$, et al. FOLFIRINOX versus gemcitabine for metastatic pancreatic cancer. N Engl J Med 2011;364:1817-25.

28. Von Hoff DD, Ervin T, Arena FP, et al. Increased survival in pancreatic cancer with nab-paclitaxel plus gemcitabine. N Engl J Med 2013;369:1691-703. 